\title{
PROPOSIÇÃO DE UMA NOVA HEURÍSTICA DE SEQUENCIAMENTO BASEADA NO ATC PARA APLICAÇÃO EM AMBIENTE FARMACÊUTICO COM TAREFAS
}

\section{A NEW ATC-BASED SCHEDULING HEURISTIC FOR PHARMACEUTICAL PRODUCTION WITH COMPLEX TASKS}

\author{
Thiago Franzen Aydos ${ }^{1}$; Michel José Anzanello ${ }^{2}$ \\ ${ }^{1}$ Universidade Federal de Santa Catarina - UFSC - Florianópolis/SC - Brasil \\ thiago.aydos@gmail.com \\ ${ }^{2}$ Universidade Federal do Rio Grande do Sul - UFRGS - Porto Alegre /RS - Brasil \\ michel.anzanello@gmail.com
}

\begin{abstract}
Resumo
A aplicação das ferramentas e princípios Lean na preparação de setups e no sequenciamento da produção faz com que empresas consigam executar tarefas em ambientes mais dinâmicos, com maior variação de mix produtivo, redução de estoques e maior efetividade no atendimento aos prazos. Para atender aos objetivos da Manufatura Enxuta através da programação da produção, este artigo propõe uma nova regra de sequenciamento derivada do Apparent Tardiness Cost (ATC) chamada Apparent Tardiness Cost with Task Complexity (ATCTC). A regra visa reduzir a concentração de tarefas de mesma complexidade no ordenamento sem incorrer em atrasos de entrega. A pesquisa caracteriza-se como aplicada, quantitativa e de caráter experimental, visando desenvolver a adaptação de uma regra de sequenciamento multicriterial já existente para o setor farmacêutico. $O$ ATCTC foi aplicado em uma empresa do setor e simulações foram realizadas em ambiente virtual para verificar o seu comportamento em distintas configurações de cenário produtivo. Os resultados obtidos comprovam a eficácia da nova heurística e sugerem significativo potencial para pesquisas futuras.
\end{abstract}

Palavras-chave: sequenciamento; complexidade de tarefa; heurísticas.

\section{Introdução}

Técnicas de sequenciamento da produção têm sido amplamente utilizadas na busca por arranjos de atividades mais eficientes, com menos perdas e mais produtividade. A utilização dessas técnicas é frequentemente viabilizada em ambientes computacionais através de pequenos softwares ou sistemas integrados de gestão mais amplos. Muitas vezes, a utilização dessas ferramentas informatizadas acaba gerando desconhecimento sobre o funcionamento da heurística que está por trás, fornecendo os resultados numéricos sem a devida adaptação das condições ao ambiente de aplicação. Um profundo entendimento dessas técnicas pode proporcionar oportunidades de aplicação inovadoras e com melhores resultados. Para solucionar os desafios da programação da 
produção, diversas heurísticas de sequenciamento foram criadas, dentre as quais destacam-se o SPT (Shortest Processing Time) (LAWRENCE; SEWELL, 1997) e o Branch and Bound (BRAH; HANSUCKER, 1991). Segundo Ichmura (1997), em um ambiente de inovações tecnológicas constantes, com diversidade de demanda e intensificação da competitividade, torna-se necessário diminuir os lotes de peças e adotar um sistema estruturado de sequenciamento da produção, de modo a ordená-la conforme a demanda, em quantidades limitadas e com menor lead time.

A fim de reduzir tempos de processamento e lead time, as empresas frequentemente recorrem a soluções custosas sem avaliar as ferramentas e princípios da manufatura enxuta na sua plenitude. $\mathrm{O}$ uso de horas extras e o investimento em capacidade produtiva são algumas alternativas que poderiam ser evitadas, muitas vezes, apenas melhorando o balanceamento e a programação da produção. Uma agenda organizada a partir de datas de entrega, com constante entrada de novas tarefas de prioridades distintas, tende a exigir uma maior capacidade de controle das variáveis envolvidas a fim de evitar atrasos ou gastos excessivos. Segundo Pinedo (2008), um dos objetivos do sequenciamento da produção é a melhor utilização dos recursos da planta, de forma a reduzir a utilização de horas extras e aumentar a produtividade. Acredita-se que, com uma programação eficiente e dinâmica da produção, é possível melhorar a eficiência dos processos e cumprir com os prazos sem artifícios indesejados.

Buscando atender à demanda por soluções de sequenciamento no processamento de produtos hospitalares em uma indústria farmacêutica, este artigo propõe uma regra baseada na heurística Apparent Tardiness Cost (ATC), tendo como principal função objetivo reduzir o tempo total de atraso. Wisner e Siferd (1995) afirmam que atender aos prazos de entrega é o objetivo mais importante da programação e apenas $58 \%$ dos gestores industriais trabalham para responder a essa demanda. Por ser tratar de uma regra com característica multicriterial, o ATC é amplamente indicado para ambientes complexos com diversidade significativa de variáveis. Este estudo se propõe a realizar uma revisão bibliográfica sobre o ATC e apontar as regras mais relevantes a ele associadas, a fim de auxiliar no desenvolvimento de uma nova heurística que reduza os tempos de atraso e considere a complexidade das tarefas. A nova regra, chamada de Apparent Tardiness Cost with Task Complexity (ATCTC), foi desenvolvida para atender às demandas do setor farmacêutico e seus resultados foram verificados a partir da aplicação em uma empresa fabricante de medicamentos hospitalares. A heurística tem como função objetivo reduzir o tempo total de atraso, buscando restringir a repetição de tarefas de mesma complexidade.

Este artigo está organizado conforme descrito a seguir. A seção 2 apresenta o referencial teórico acerca das principais regras do ATC. A seção 3 apresenta a metodologia utilizada para o desenvolvimento da regra e para a validação dos resultados. Na seção 4 as etapas de desenvolvimento do ATCTC serão descritas detalhadamente, descrevendo a nova regra. A seção 5 
apresenta os resultados provenientes do desenvolvimento da regra, bem como a aplicação de simulação para avaliar as características da nova regra em cenários variados. Por fim, a seção 6 descreve as conclusões do artigo.

\section{Referencial teórico}

Os princípios da Manufatura Enxuta (Lean Manufacturing) passam a ser cada vez mais disseminados entre os gestores de operações, buscando reduzir as perdas em geral e aumentar os ganhos de um sistema produtivo. Pode-se definir Manufatura Enxuta como uma abordagem que busca uma melhor maneira de organizar e gerenciar a cadeia de suprimentos, o desenvolvimento de produtos, a operação da produção e os relacionamentos de uma empresa com seus clientes, buscando fazer cada vez mais com menos recursos e em menor tempo (WOMACK; JONES, 1996).

O sequenciamento da produção constitui um campo de estudo estratégico para a administração de sistemas de manufatura, influenciando significativamente na busca pelos pilares da manufatura enxuta. Segundo Pinedo (2008), sequenciamento é a alocação de recursos destinados a tarefas em um determinado período de tempo, visando otimizar um ou mais objetivos. Esses recursos e tarefas são organizados de diversas formas, podendo representar, por exemplo, máquinas em um chão de fábrica, pistas de pouso em um aeroporto, pontes rolantes em um canteiro de obras e também unidades de processamento em um ambiente computacional. Buscar arranjos de sequenciamento a partir de métodos científicos pode trazer benefícios que, muitas vezes, são de fácil percepção para o gestor.

O sequenciamento da produção e as práticas Lean tem seu valor cada vez mais reconhecido no mundo empresarial, ultrapassando as fronteiras da manufatura tradicional e se propagando por segmentos diversificados, como o da gestão pública, das finanças e do setor farmacêutico. Segundo Papavasileiou et al. (2007), a competição global na indústria farmacêutica e o aumento da demanda por medicamentos a preços mais acessíveis por governos e cidadãos tem atraído a atenção da indústria para eficiência produtiva. Altria et al. (2009) afirmam que muitos fornecedores do setor farmacêutico têm implementado práticas Lean para reduzir as perdas, custos, tempos de ciclo e variabilidade na produção. $\mathrm{Na}$ administração da produção, é possível mensurar o custo do esforço despendido para a maioria das tarefas realizadas, possibilitando a identificação das melhorias geradas pelo sequenciamento. Reduzir tempos de execução e melhorar o aproveitamento dos recursos pode gerar ganhos financeiros reais, proporcionando um melhor gerenciamento dos turnos de trabalho e criando oportunidades de melhoria geral em um ambiente de manufatura.

Técnicas de programação da produção buscam otimizar as funções objetivo que são estabelecidas a partir da relação direta com o contexto produtivo (SLACK et al., 2002). A ordenação das tarefas pode ser feita a partir da identificação das necessidades do cliente, que são 
priorizadas conforme seu grau de importância. Nesse contexto, podemos citar alguns exemplos de situações a serem priorizadas, como em contextos de emergência, quando há insatisfação crítica do cliente ou em casos de prioridades preestabelecidas por contrato. É possível ainda classificar tarefas a partir dos prazos de entrega dos clientes, sendo o trabalho sequenciado de acordo com as datas acordadas, de forma a atender ao máximo de encomendas possível ou minimizar o tempo total de atraso. Também pode-se priorizar sequenciamento a partir do tempo de trabalho a ser realizado, reduzindo-se o tempo total gasto em um processo ou ao minimizar-se o tempo ocioso dos centros produtivos.

Empresas do setor farmacêutico operam em ambientes complexos, onde processos envolvem múltiplas etapas inter-relacionadas que usam inúmeros materiais de diferentes fornecedores (ARASH et al., 2013). Para resolver problemas de sequenciamento em ambientes de nível de complexidade elevado, são utilizados métodos computacionais chamados de heurísticas ou algoritmos. Este estudo aborda a aplicação da heurística Apparent Tardiness Cost (ATC), desenvolvida por Rachamadugu e Morton (1982), com vistas à minimização do tempo total de atraso em um grupo de tarefas com datas de entrega e tempos de processo definidos. Como exposto a seguir, essa heurística vem sofrendo modificações com o intuito de adequá-la à realidade produtiva nos mais variados cenários. De forma genérica, o problema do ATC é formado por um grupo de $n$ tarefas a serem processadas por uma ou mais máquinas. Cada tarefa possui as seguintes variáveis: tempo de processamento $\left(p_{j}\right)$, que é o tempo contado desde o início até o final da realização da tarefa; a média dos tempos de processamento $\left(p_{j} m e d\right)$, que se dá pela média aritmética dos tempos de processamentos selecionados; um tempo de entrega $\left(d_{j}\right)$, que é o tempo limite no qual a tarefa deve ser realizada ou entregue; um peso ou penalidade $\left(w_{j}\right)$, que deve ser estimado a partir de fatores de priorização ou penalidades por atraso na entrega da tarefa; um instante de liberação $\left(r_{j}\right)$, que consiste no período de tempo que a tarefa deve aguardar até o início da sua execução. Para as tarefas subsequentes à tarefa executada, é considerado o tempo de setup $\left(s_{i j}\right)$, que consiste no tempo necessário para ajustar as condições de execução da tarefa na espera, ou seja, é o tempo que precede a sua execução. Por fim, a heurística do ATC pode utilizar parâmetros de escala $k$, também conhecidos como parâmetros look-ahead, obtidos de forma empírica de acordo com o sistema em análise.

Rachamadugu e Morton (1982) propuseram a regra do ATC a fim de minimizar o tempo total de atraso em uma máquina única. A regra integra um termo associado ao tempo de processamento com um termo de folga, utilizando a variável de data de entrega da tarefa, conforme a equação (1). O primeiro termo, chamado de Termo de Priorização, atribui às tarefas com alta prioridade $w_{j}$ ou baixo tempo de processamento $p_{j}$ um índice elevado. O segundo termo é chamado 
de Termo de Folga, o qual prioriza tarefas com data de entrega mais próxima e quando o instante de liberação não for próximo.

$$
\operatorname{ATC}(t, j)=\frac{w j}{p j} * \exp \left(-\frac{\max (d j-\mathrm{rj}-t, 0)}{k^{1} * \text { pjmed }}\right)
$$

Zhang et al. (2012) utilizaram a regra do ATC para propor um modelo de sequenciamento em sistemas Job Shop com três funções objetivo, a partir da análise dos problemas enfrentados no setor automotivo. Segundo Jensen et al. (1995), o ATC é a heurística de sequenciamento mais efetiva para minimizar o tempo total de atraso. Lamothe et al. (2012) afirmam que a regra é conhecida por ser eficiente em modelos com máquinas para reduzir tempos de atraso, e propõem duas extensões da regra: o ATCOEE (Apparent Tardiness Cost with Overall Equipment Effectiveness) e o ATCOEEF (Apparent Tardiness Cost with Overall Equipment Effectiveness and Flexiblity). Valente et al. (2012) defendem que estudos computacionais mostram o ATC como uma das heurísticas com melhor desempenho no atendimento da função objetivo, considerando as regras existentes para problemas lineares.

A partir da regra básica do ATC, vários pesquisadores desenvolveram aprimoramentos de forma a incluir variáveis que influenciassem na busca pela melhor solução de sequenciamento. Esses aprimoramentos são as chamadas ATC-Based Rules. A regra do Apparent Tardiness Cost with Setups (ATCS) considera sequenciamento com setups dependentes, ou seja, leva em conta o tempo de preparação que antecede o início de uma determinada atividade. Segundo Lee et al.(1997), o ATCS é a regra mais compacta dentre as regras baseadas no ATC que agregam o termo de setup $S_{i j}$ entre duas atividades, como é possível ver na equação (2).

$$
\operatorname{ATCS}(t, i, j)=\frac{w j}{p j} * \exp \left(-\frac{\max (d j-\mathrm{rj}-t, 0)}{k^{1} * \text { pjmed }}\right) * \exp \left(-\frac{S i j}{k^{2} * \text { Smed }}\right)
$$

Onde: $i=$ última tarefa programada; $S_{i j}=$ tempo de setup entre duas atividades e Smed= tempo médio de setup de todas as atividades

Park et al. (2000) realizaram um estudo de sequenciamento de tarefas em máquinas paralelas e propuseram uma extensão do ATCS que utiliza parâmetros look-ahead para calcular o índice de prioridades de cada tarefa. Embasados na regra do ATCS, Pfund et al. (2008) propuseram a regra Apparent Tardiness Cost with Setups and Ready times (ATCSR), a fim de solucionar o problema de sequenciamento, considerando os tempos de liberação de tarefas em máquinas paralelas idênticas com setups dependentes da sequência. Trata-se de uma das mais efetivas regras de sequenciamento, visto que permite solucionar problemas dinâmicos com setups dependentes da sequência e do instante de liberação. A expressão é apresentada na equação (3). 


$$
\operatorname{ATCSR}(t, i, j)=\frac{w j}{p j} * \exp \left(-\frac{\max (d j-\mathrm{rj}-t, 0)}{k^{1} * \text { pjmed }}\right) * \exp \left(-\frac{s i j}{k^{2} * s m e d}\right) * \exp \left(-\frac{\max (r j-t, 0)}{k^{3} * \text { pjmed }}\right)
$$

Driessel et al. (2010) utilizam ATCSR como principal benchmarking de comparação para avaliar o desempenho das variantes do método de busca de variáveis "Variable Neighborhood Search" (VNS) (Mladenovic e Hansen, 1997) em máquinas paralelas, onde citam sua aplicabilidade no setor de produção de semicondutores.

Xi e Jang (2012) propuseram duas novas extensões do ATC. O Modified Aparent Tardiness Cost with Setups and Ready Times (MATCSR) foi proposto para solucionar o problema em uma máquina única com tempos de liberação futuros e setups dependentes de sequência contínua. Nesta regra, descrita pela equação (4), o primeiro termo inclui o tempo de setup dependente da sequência e o tempo desde a tomada de decisão até o instante de liberação da tarefa, de forma a evitar que tarefas com menor instante de liberação sejam prorrogadas.

$$
\begin{aligned}
\operatorname{MATCSR}(t, i, j)= & \frac{w j}{p j+\operatorname{sij}+\max (r j-t, 0)} * \exp \left(-\frac{\max (d j-p j-\operatorname{sij}-\max (r j, t), 0)}{k^{1} * \text { pjmed }}\right) * \exp \left(-\frac{s i j}{k^{2} * s m e d}\right) \\
& * \exp \left(-\frac{\max (r j-t, 0)}{k^{3} * \text { pjmed }}\right)
\end{aligned}
$$

A segunda extensão proposta por Xi e Jang (2012), chamada de Apparent Tardiness Cost with Separable Setup and Ready Time (ATCSSR), visa liberar uma atividade somente após o setup. Quando uma tarefa é finalizada, ambas as regras selecionam outra em espera para ser processada a seguir. O MATCSR permite que a máquina permaneça disponível antes da preparação do setup, enquanto o ATCSSR permite que a máquina esteja disponível somente após o setup. Um exemplo disso é quando o setup de uma tarefa selecionada é iniciado no tempo de decisão e pode ser finalizado antes que a tarefa esteja liberada para processamento. O ATCSSR é apresentado na equação (5).

$$
\begin{aligned}
\operatorname{ATCSSR}(t, i, j)= & \frac{w j}{p j+\max (s i j, r j-t)} * \exp \left(-\frac{\max (d j-p j-\max (r j, t+s i j), 0)}{k^{1} * \text { pjmed }}\right) * \exp \left(-\frac{s i j}{k^{2} * s m e d}\right) \\
& * \exp \left(-\frac{\max (r j-t-s i j, 0)}{k^{3} * \text { pjmed }}\right)
\end{aligned}
$$

Em testes de comparação de regras para ambientes com setup contínuo e separado, as regras do MATCSR e ATCSSR superam a regra ATCSR. A Tabela 1 apresenta as cinco regras de ATC citadas na revisão deste trabalho. Elas foram organizadas em ordem cronológica, e os termos de cada regra foram separados e agrupados nas colunas da tabela. Dessa forma, é possível observar como as regras baseadas no ATC se alteram ao longo do tempo, utilizando-se dos elementos das versões anteriores para reduzir o tempo total de atraso. 
Tabela 1 - Regras baseadas no ATC

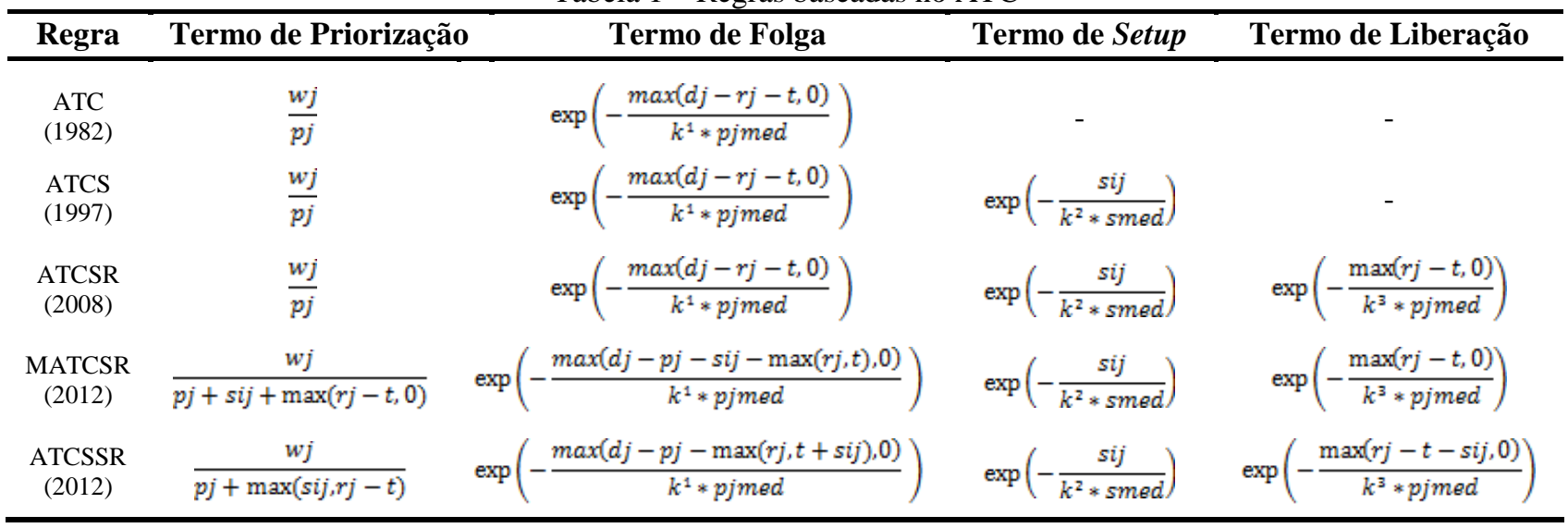

Fonte: Adaptado de Pfund et al. (2008)

\section{Método}

O método utilizado neste trabalho tem como objetivo desenvolver e avaliar uma nova regra de sequenciamento de tarefas para o setor farmacêutico, com base nas heurísticas de ATC previamente revisadas. A pesquisa realizada caracteriza-se como aplicada, quantitativa e de caráter experimental, visando desenvolver a adaptação de uma regra de sequenciamento multicriterial já existente para o cenário farmacêutico e verificar os resultados da sua aplicação.

Para definir as funções objetivo da nova regra, uma análise das características do ambiente de aplicação foi realizada. A partir da análise das regras já existentes, os termos que compõem a nova regra foram determinados, formando a composição da heurística chamada Apparent Tardiness Cost with Task Complexity (ATCTC). Com a expressão já estruturada, um breve estudo de calibração dos parâmetros dos termos foi realizado. De forma a validar e avaliar o desempenho da nova regra, uma aplicação no cenário farmacêutico foi feita, realizando sequenciamento da produção de uma empresa com o ATC e com o ATCTC. Dessa forma, foi possível avaliar e comparar os resultados entre as duas heurísticas. Por fim, uma simulação foi feita para avaliar características da nova regra, como robustez e flexibilidade. Nessa simulação, o ATC e o ATCTC foram testados paralelamente em 12 cenários distintos com 200 simulações para cada cenário, onde foram variados os parâmetros de data de entrega, média de tempo de processamento $\left(p_{j} m e d\right)$ e desvio padrão (Desvpad $p_{j}$ ) da distribuição normal dos tempos de processamento. $\mathrm{O}$ ambiente computacional escolhido para simulação foi o software Microsoft Excel, com o auxílio de programação em Visual Basic.

As etapas do método proposto são apresentadas na Figura 1 e detalhadas na seção 4. 


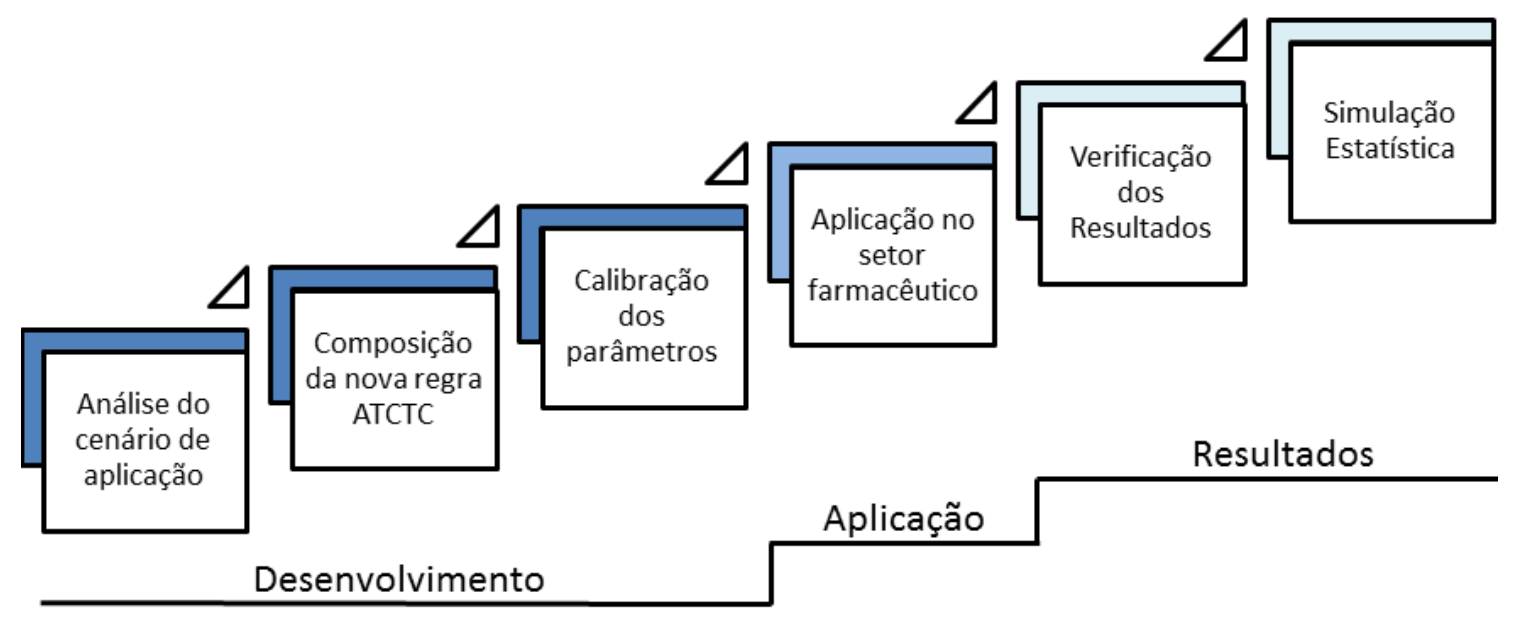

Fonte: Autoria própria (2015)

\section{Desenvolvimento do Apparent Tardiness Cost with Task Complexity (ACTCT)}

\subsection{Análise do cenário de aplicação}

A aplicação deste estudo realizou-se em uma empresa do setor farmacêutico que fornece produtos hospitalares. A empresa produz bolsas de até 5 litros com soluções químicas para tratamento de pacientes em tratamento. Está situada em Porto Alegre, conta com 30 funcionários diretos e possui um portfólio de 25 produtos com variações de especificações técnicas relevantes, como composição química, volume e prazos de entrega. $\mathrm{O}$ cumprimento das normas regulamentadoras de segurança e qualidade e o atendimento aos prazos de entrega são aspectos de extrema importância para a satisfação dos clientes. A empresa realiza o gerenciamento da programação de forma empírica, com o auxílio de fichas de controle de prazos.

A partir da análise do portfólio de produtos da empresa, identificaram-se características que influenciam significativamente no processo de sequenciamento. Primeiramente, as datas de entrega $\left(d_{j}\right)$ possuem a prioridade mais elevada e devem ser atendidas com rigor pelos motivos descritos anteriormente. Os tempos de processamento $\left(p_{j}\right)$ são conhecidos. Não há sistemática de priorização de consumidores por parte da empresa, mas sim uma prioridade de famílias de produtos, as quais são produzidas sob encomenda e não podem atrasar. Outro fator a ser considerado é o de repetitividade do processo, que muitas vezes implica em prejuízos físicos para os operadores. Diversas matérias-primas possuem peso elevado, e o seu manuseio por muito tempo de forma ininterrupta pode causar danos físicos e doenças de esforço repetitivo. A complexidade da composição dos produtos também deve ser considerada. É sabido que intercalar produtos com diferentes especificações ou complexidades pode diminuir a repetitividade nos processos, reduzindo a probabilidade de falhas de operação e prevenindo doenças relacionadas ao trabalho por esforço 
repetitivo (Strasser, 1995). Dessa forma, uma das restrições da nova regra deve ser o controle da alternância de tarefas, de acordo com suas complexidades.

A fim de ajustar a configuração do cenário de aplicação dentro das premissas características do sequenciamento, deve-se adequar a forma de visualização do sistema produtivo farmacêutico. As salas de trabalho serão consideradas como máquinas idênticas em paralelo, com setup dependente da sequência. O instante de liberação das tarefas será considerado 0 (zero) para todos os produtos. Os parâmetros look-ahead também possuem valor igual a 1 (um), pois acredita-se que é mais adequado equilibrar o peso dos termos com os coeficientes naturais advindos da configuração do cenário. Por se tratar de um sistema de produção onde cada operador é encarregado de todas as etapas de manufatura, os postos de trabalho são considerados como máquinas únicas com mesmo tempo de processamento e de setup.

Dados da programação da produção da empresa foram coletados e uma comparação foi realizada utilizando as heurísticas ATC e ATCTC. O período de produção programado é de dez dias. Os tempos de processamento variam de duas a três horas para preparação e execução das tarefas. Cada tarefa possui uma data de entrega e uma família de complexidade. No cenário em questão, produtos de apenas dois grupos de complexidade são considerados: tipos 1 e 2. Para ambas as regras, atribui-se o valor para a constante $k_{1}=1$. Para a constante de complexidade, conforme explicado anteriormente, atribuiu-se o valor de $K c=2$. Como a empresa não possui priorização de clientes ou famílias de produtos, o parâmetro $w_{j}$ também terá o valor igual a 1 (um).

\subsection{Composição do Apparent Tardiness Cost with Task Complexity (ATCTC)}

A nova regra é uma formulação baseada na heurística do ATC e, portanto, tem como principal função objetivo reduzir o tempo total de atraso. Após a análise do cenário de aplicação, verifica-se a necessidade de inserir uma restrição relacionada à complexidade das tarefas, de forma que não se repitam tarefas similares. O primeiro termo a ser adicionado na composição da regra é o termo de priorização $\left(\frac{w j}{p j}\right)$. O termo de folga também é adicionado, baseando-se na regra original do ATC, conforme a expressão $\exp \left(-\frac{\max (d j-r j-t, 0)}{k^{1} * \text { pjmed }}\right)$.

O terceiro termo é chamado de termo de Complexidade, dado pela expressão $\exp \left(-\frac{\left(N^{o} \operatorname{Rep}\right)}{K c *(K c+T a j)}\right)$, e constitui uma contribuição original da pesquisa. Este termo tem como principal objetivo alternar tarefas de diferentes níveis de complexidade, ou seja, a regra não permitirá que tarefas similares sejam ordenadas em sequência, a menos que se encontrem em situação de atraso. O termo é composto da seguinte forma: o numerador contabiliza o número de repetições que ocorrem quando tarefas de mesma complexidade são inseridas em sequência $\left(N^{o}\right.$ Rep $)$. Dessa forma, quanto maior o valor de $N^{o} R e p$, menor será o índice da tarefa a ser alocada. 
No denominador, aparecem duas variáveis distintas: uma Constante de Complexidade $(K c)$ e uma variável de Tempo de Atraso $(\mathrm{Ta})$. A constante de complexidade é usada para definir o grau de impacto que o termo terá no índice de ordenamento da tarefa. Quanto menor $K c$, maior será o impacto do termo na composição do índice de ordenamento. O valor de $K c$ é obtido de forma empírica, como demonstrado no capítulo 4.3.

A variável de Tempo de Atraso $\left(T a_{j}\right)$ visa reduzir a penalização das tarefas que já estiverem com atraso de entrega. O Tempo de Atraso $\left(T a_{j}\right)$ indica o número total de horas de atraso após o processamento da tarefa, conforme a equação: $T a_{j}=d_{j}-r_{j}-p_{j}$. Com a inclusão do tempo de atraso no denominador, penaliza-se de forma menos rigorosa as tarefas que estejam atrasadas. Assim, a alternância de complexidades não impactará significativamente no tempo total de atraso. O denominador foi multiplicado pela constante $K c$ para regular o seu impacto sobre o valor final do termo, atribuindo maior significância à constante.

A expressão do ATCTC é apresentada na equação (6):

$$
\operatorname{ATCTC}(t, j)=\frac{w j}{p j} * \exp \left(-\frac{\max (d j-\mathrm{rj}-t, 0)}{k^{1} * \text { pjmed }}\right) * \exp \left(-\frac{\left(N^{o} \operatorname{Rep}\right)}{K c *(K c+T a j)}\right)
$$

\subsection{Calibração dos parâmetros}

Detalhes acerca da definição da constante $K c$ são apresentados nessa seção. Para identificar um valor apropriado para a constante, simulações com diferentes valores de $K c$ foram realizadas, avaliando-se o valor final do Termo de Complexidade e aproximando um valor mais adequado para a constante. Nessa análise, foram consideradas somente tarefas isentas de atrasos, com $T a=0$. A Tabela 2 apresenta os valores correspondentes ao termo de complexidade de acordo com a variação de $K c$.

Tabela 2 - Valores finais do Termo de complexidade para diferentes valores de Kc

\begin{tabular}{ccccccccccc}
\hline Kc & \multicolumn{1}{c}{} & \multicolumn{1}{c}{$\mathbf{N}^{\mathbf{0}}$ de Repetições de Complexidade } & $\mathbf{9}$ & $\mathbf{9}$ \\
\hline & $\mathbf{1}$ & $\mathbf{2}$ & $\mathbf{3}$ & $\mathbf{4}$ & $\mathbf{5}$ & $\mathbf{6}$ & $\mathbf{7}$ & $\mathbf{8}$ & $\mathbf{9}$ \\
\hline 1 & 0,3679 & 0,1353 & 0,0498 & 0,0183 & 0,0067 & 0,0025 & 0,0009 & 0,0003 & 0,0001 & 0,0000 \\
2 & 0,7788 & 0,6065 & 0,4724 & 0,3679 & 0,2865 & 0,2231 & 0,1738 & 0,1353 & 0,1054 & 0,0821 \\
3 & 0,8948 & 0,8007 & 0,7165 & 0,6412 & 0,5738 & 0,5134 & 0,4594 & 0,4111 & 0,3679 & 0,3292 \\
4 & 0,9394 & 0,8825 & 0,8290 & 0,7788 & 0,7316 & 0,6873 & 0,6456 & 0,6065 & 0,5698 & 0,5353 \\
\hline
\end{tabular}

Fonte: Autoria própria (2015)

A tabela mostra que, quanto maior o valor de $K c$, menor será a penalização da tarefa. Para realizar a escolha do valor, foram considerados dois aspectos: a quantidade de repetições aceitáveis no cenário a ser simulado e o grau de penalização que o termo deve atribuir no ordenamento da tarefa. No cenário em questão, é desejável que tarefas de mesma complexidade não sejam colocadas em sequência. Para tanto, a penalidade deve ser razoável desde a primeira repetição. Ao definir 
$K c=2$, penaliza-se a tarefa repetida de forma adequada, atribuindo ao índice da tarefa o valor de $77,8 \%$ do seu número original já na primeira repetição. Os valores de penalização são distribuídos de forma satisfatória entre as seis primeiras repetições, dando assim estabilidade ao funcionamento do termo e adequando-se ao cenário de aplicação.

Para testar a eficácia dessa combinação, verificou-se o impacto do valor da variável no Termo de Complexidade com quantidades de horas de atraso distintas, utilizando $K c$ com diversos valores. Os resultados são apresentados na Tabela 3, utilizando-se a constante $K c=2$.

Tabela 3 - Valores finais do Termo de complexidade para diferentes valores de Ta

\begin{tabular}{ccccccc}
\hline Ta & \multicolumn{7}{c}{$\mathbf{N}^{\mathbf{0}}$ de Repetições de Complexidade } \\
\hline 5 & $\mathbf{1}$ & $\mathbf{2}$ & $\mathbf{3}$ & $\mathbf{4}$ & $\mathbf{5}$ & $\mathbf{6}$ \\
\hline 15 & 0,3679 & 0,1353 & 0,0498 & 0,0183 & 0,0067 & 0,0025 \\
30 & 0,9817 & 0,9636 & 0,9460 & 0,9286 & 0,9116 & 0,8948 \\
45 & 0,9899 & 0,9800 & 0,9702 & 0,9604 & 0,9507 & 0,9412 \\
\hline
\end{tabular}

Fonte: Autoria própria (2015)

Os resultados confirmam que o Termo de Complexidade diminui a penalização das tarefas à medida que o valor do tempo de atraso aumenta. Portanto, é possível evitar a alocação de tarefas de mesma complexidade em sequência sem comprometer a função objetivo principal, que é reduzir o tempo total de atraso.

\section{Aplicação do ATCTC no setor farmacêutico}

\subsection{Verificação dos resultados numéricos}

A Tabela 4 apresenta o resultado da programação com as duas heurísticas, especificando a ordem de produção, o código e a complexidade da tarefa e o tempo de atraso após a sua realização. Nos resultados referentes ao ATCTC, as linhas em negrito indicam que a ordem foi alterada em relação ao ordenamento do ATC.

Tabela 4 - Comparação das sequências geradas pelas regras ATC e ATCTC.

\begin{tabular}{ccccccc}
\hline & \multicolumn{2}{c}{ ATC } & & \multicolumn{3}{c}{ ATCTC } \\
\hline Ordem & Código Tarefa & Complex & Atraso (h) & Código Tarefa & Complex & Atraso (h) \\
\hline 1 & 2 & 2 & 0,00 & 2 & 2 & 0,00 \\
2 & 17 & 2 & 0,00 & 17 & 2 & 0,00 \\
3 & 4 & 1 & 0,00 & 4 & 1 & 0,00 \\
4 & 18 & 2 & 0,00 & 18 & 2 & 0,00 \\
5 & 30 & 2 & 0,00 & $\mathbf{1 5}$ & $\mathbf{1}$ & $\mathbf{0 , 0 0}$ \\
6 & 15 & 1 & 0,00 & $\mathbf{3 0}$ & $\mathbf{2}$ & $\mathbf{0 , 0 0}$ \\
7 & 16 & 1 & 1,09 & 16 & 1 & 1,09 \\
8 & 10 & 1 & 0,00 & $\mathbf{2 1}$ & $\mathbf{2}$ & $\mathbf{0 , 0 0}$ \\
9 & 3 & 1 & 0,00 & $\mathbf{1 0}$ & $\mathbf{1}$ & $\mathbf{0 , 0 0}$ \\
10 & 21 & 2 & 0,00 & $\mathbf{3}$ & $\mathbf{1}$ & $\mathbf{0 , 0 0}$ \\
11 & 9 & 1 & 0,00 & 9 & 1 & 0,00
\end{tabular}




\begin{tabular}{lcccccc}
13 & 12 & 2 & 0,97 & $\mathbf{1 1}$ & $\mathbf{1}$ & $\mathbf{0 , 9 7}$ \\
14 & 24 & 1 & 0,00 & 24 & 1 & 0,00 \\
15 & 20 & 1 & 0,00 & $\mathbf{2 3}$ & $\mathbf{2}$ & $\mathbf{0 , 0 0}$ \\
16 & 23 & 2 & 2,11 & $\mathbf{2 0}$ & $\mathbf{1}$ & $\mathbf{2 , 1 1}$ \\
17 & 1 & 1 & 0,00 & 1 & 1 & 0,00 \\
18 & 13 & 1 & 0,15 & $\mathbf{2 9}$ & $\mathbf{2}$ & $\mathbf{1 , 1 6}$ \\
19 & 29 & 2 & 4,19 & $\mathbf{1 3}$ & $\mathbf{1}$ & $\mathbf{4 , 1 9}$ \\
20 & 22 & 2 & 0,00 & 22 & 2 & 0,00 \\
21 & 7 & 2 & 0,28 & 7 & 2 & 0,28 \\
22 & 8 & 2 & 2,51 & 8 & 2 & 2,51 \\
23 & 25 & 1 & 0,00 & 25 & 1 & 0,00 \\
24 & 26 & 1 & 0,00 & 26 & 1 & 0,00 \\
25 & 14 & 2 & 3,83 & 14 & 2 & 3,83 \\
26 & 19 & 1 & 0,00 & 19 & 1 & 0,00 \\
27 & 5 & 1 & 2,11 & 2 & 1 & 2,11 \\
28 & 28 & 2 & 0,00 & 6 & 2 & 0,00 \\
29 & 6 & 1 & 0,00 & 27 & 1 & 0,00 \\
30 & 27 & 1 & 2,46 & & 1 & 2,46 \\
\hline
\end{tabular}

Fonte: Autoria própria (2015)

As alterações realizadas pelo método ATCTC no ordenamento apontam um resultado mais satisfatório do que o ATC. Essas alterações não provocaram aumento significativo no tempo total de atraso das tarefas e aumentaram a alternância de complexidades em 17\%. Percebe-se que, de 28 repetições de complexidade possíveis, o ATC realizou 14 contra 10 repetições realizadas pelo ATCTC. Em valores absolutos, o ATCTC acrescentou apenas 1 hora na soma do tempo total de atraso, não causando alteração prática significativa na função objetivo.

Para verificar a eficiência do método ATCTC, foi utilizado um indicador de percentual de saturação. A saturação é dada pela concentração de repetições de tarefas de mesma complexidade em uma série de tarefas programadas pela regra, ou seja: $\operatorname{Sat}(\%)=$ "No de Repetições realizadas" / "Nº máximo de Repetições". Dessa forma, quanto maior o percentual de saturação, maior o grau de repetitividade das tarefas e menor a eficiência do algoritmo proposto. A Tabela 5 apresenta o percentual de saturação de ambos os métodos, bem como o tempo total de atraso na realização de todas as tarefas.

Tabela 5 - Percentual de saturação das regras ATC e ATCTC.

\begin{tabular}{ccc}
\hline & ATC & ATCTC \\
\hline Sat (\%) & $50 \%$ & $36 \%$ \\
Atraso (h) & 19,7 & 20,7 \\
\hline
\end{tabular}

Fonte: Autoria própria (2015)

\subsection{Simulação}

A fim de avaliar a robustez do ATCTC, realizou-se uma série de simulações em um ambiente computacional. Foram utilizados 12 cenários distintos com 200 simulações para cada cenário, comparando os métodos ATC e ATCTC em distintas configurações de produção. Cada cenário possui um calendário de 15 dias e 30 tarefas com características distintas, que variam de acordo com os parâmetros estabelecidos. Para cada parâmetro, foram selecionadas faixas de 
variação, escolhidas de acordo com as características da produção no setor farmacêutico (ver Tabela $6)$.

Tabela 6 - Parâmetros e faixas de variação

\begin{tabular}{|c|c|c|c|c|}
\hline Parâmetros & Data de Entrega & Parâmetros & $p_{i} m e d$ & Desvpad $p_{i}$ \\
\hline Folga & $130 \%$ & Alto & 4,00 & 0,88 \\
\hline Nominal & $100 \%$ & Baixo & 2,00 & 0,44 \\
\hline
\end{tabular}

Fonte: Autoria própria (2015)

Com essa variação nos parâmetros, objetiva-se avaliar a saturação do ATCTC em comparação com o ATC, especialmente nos cenários que as datas de entrega são mais apertadas. Para realizar a simulação, as datas de entrega foram calculadas de acordo com um tempo médio de processamento e de desvio padrão das tarefas. A Tabela 7 apresenta o resultado médio de todos os cenários testados, indicando o tempo total de atraso e o percentual de saturação para cada uma das heurísticas. A tabela também mostra a diferença de saturação de uma heurística para outra, de forma a entender a influência dos parâmetros no desempenho global das regras

Tabela 7 - Simulação ATC x ATCTC

\begin{tabular}{|c|c|c|c|c|c|c|c|}
\hline \multirow[b]{2}{*}{$\begin{array}{l}\text { Data de } \\
\text { Entrega }\end{array}$} & \multirow[b]{2}{*}{$p_{j}$ med $_{j}$} & \multirow[b]{2}{*}{ Desvpad $p_{j}$} & \multicolumn{2}{|c|}{ ATC } & \multicolumn{2}{|c|}{ ATCTC } & \multirow[b]{2}{*}{$\triangle \mathrm{SAT}$} \\
\hline & & & $\operatorname{Atraso}(\mathbf{h})$ & SAT \% & $\operatorname{Atraso}(\mathbf{h})$ & SAT \% & \\
\hline Folga & Alta & Alta & 0,0 & $43 \%$ & 0,0 & $14 \%$ & $30 \%$ \\
\hline Folga & Alta & Baixa & 0,0 & $43 \%$ & 0,0 & $9 \%$ & $34 \%$ \\
\hline Folga & Baixa & Alta & 0,7 & $42 \%$ & 0,7 & $21 \%$ & $21 \%$ \\
\hline Folga & Baixa & Baixa & 0,0 & $44 \%$ & 0,0 & $14 \%$ & $30 \%$ \\
\hline Nominal & Alta & Alta & 17,2 & $42 \%$ & 17,4 & $14 \%$ & $28 \%$ \\
\hline Nominal & Alta & Baixa & 15,7 & $43 \%$ & 15,8 & $9 \%$ & $34 \%$ \\
\hline Nominal & Baixa & Alta & 2,3 & $42 \%$ & 2,3 & $21 \%$ & $21 \%$ \\
\hline Nominal & Baixa & Baixa & 0,0 & $43 \%$ & 0,0 & $14 \%$ & $29 \%$ \\
\hline Reduzida & Alta & Alta & 441,6 & $42 \%$ & 442,2 & $32 \%$ & $10 \%$ \\
\hline Reduzida & Alta & Baixa & 457,4 & $43 \%$ & 457,9 & $29 \%$ & $15 \%$ \\
\hline Reduzida & Baixa & Alta & 2,6 & $42 \%$ & 2,6 & $21 \%$ & $21 \%$ \\
\hline Reduzida & Baixa & Baixa & 0,0 & $43 \%$ & 0,0 & $14 \%$ & $28 \%$ \\
\hline
\end{tabular}

Fonte: Autoria própria (2015)

Os resultados mostram que o ATCTC reduziu a incidência de tarefas de mesma complexidade em sequência em até $34 \%$, sem que isso ocasionasse aumento significativo no tempo total de atraso. Verifica-se ainda que, nos cenários onde houve maior tempo de atraso, a heurística do ATCTC registrou percentuais de variação de saturação superiores aos cenários normais, não sacrificando prazos de entrega ao alternar a complexidade das tarefas ordenadas e reduzir a saturação. A regra do ATC demonstrou desempenho similar para todos os casos, registrando, em média, $43 \%$ de saturação, ou seja, realizou um pouco mais da metade das alternâncias possíveis na programação das tarefas, obtendo melhores resultados com relação ao tempo total de atraso do que o ATCTC. Outra constatação interessante é que a regra do ATCTC proporciona maior saturação para tempos de processamento organizados em uma distribuição normal com menor desvio padrão. 
A utilização do Microsoft Excel combinada com programação em Visual Basic se mostrou uma alternativa rápida, flexível e com boa interface gráfica para simulação. O tempo total de processamento referente às 2.400 rodadas da simulação completa não ultrapassou 15 minutos, o que viabilizou a repetição do processo sempre que necessário.

\section{Conclusão}

A fim de encontrar uma solução para a programação da produção que evite repetições de tarefas de mesma complexidade, este estudo propôs a heurística chamada ATCTC, que é uma derivação da conhecida regra do ATC. Visando atender a programação de tarefas com baixo percentual de repetição (Sat \%), o ATCTC obteve saturação menor que o ATC em todos os cenários testados. Os resultados da aplicação na empresa farmacêutica mostraram que o percentual de saturação do algoritmo proposto foi, em média, $25 \%$ mais baixo do que o original. Conforme proposto, verificou-se que o ATCTC respeita a função objetivo da heurística em todas as situações de atraso real, não causando prejuízos significativos de tempo total de atraso ao realizar o ordenamento das tarefas.

A busca pela excelência na preparação de setups e na troca rápida de ferramentas possibilita executar tarefas com mix de produção dinâmico, reduzindo estoques e atendendo prazos mais justos. Para auxiliar nas demandas do Lean Manufacturing e do seu pilar Just in Time, os autores propõem novos estudos do ATCTC adicionando termos de regras derivadas do ATC já conhecidos, tais como o termo de setup e o termo de liberação de tarefas. Por se tratar de uma heurística altamente flexível, acredita-se que há um grande potencial para a sua exploração e adaptação em ambientes mais complexos.

Também se sugere um estudo mais aprofundado sobre a determinação da Constante de Complexidade $(K c)$, identificando faixas de utilização de acordo com o grau de impacto desejado da complexidade das tarefas sobre o ordenamento final.

\footnotetext{
Abstract

The application of Lean tools and principles in machine setups preparation and production scheduling activities helps companies to execute tasks in dynamic environments, with variation of production mix, with inventory reduction and meeting deadlines in a effectively way. In order to meet the goals of Lean Manufacturing, this paper proposes a new dispatching rule derived from the Apparent Tardiness Cost (ATC) called Apparent Tardiness Cost with Task Complexity (ATCTC). This rule aims to reduce sequencing tasks with identical complexity levels without yielding delays in task delivery. The research is characterized as applied, quantitative and experimental, in order to develop an adaptation of a multi-criteria sequencing rule for the pharmaceutical segment. The ATCTC was applied in a pharmaceutical company and simulations have been executed in a virtual environment to verify its robustness under different parameters and scenarios. The results
} 
corroborated the effectiveness of the new heuristic and suggested significant potential for future research.

Key-words: scheduling; task complexity; heuristics.

\section{Referências}

ALTRIA, K.; DUFTON, A.; CARLEYSMITH, S. Learning from lean sigma. Pharmaceutical Technology Europe, v. 21, n. 2, p. 16-24, 2009. crossref

ARASH, A.; PATEL, P. C.; ZANGOUEINEZHAD, A.; LINDERMAN, K. The effect of environmental complexity and environmental dynamism on lean practices. Journal of Operations Management, v. 31, n. 4, p. 193-212, 2013.

BRAH, S. A.; HUNSUCKER, J. L. Branch and bound algorithm for the flow shop with multiple processors. European Journal of Operations Research, v. 51, n. 1, p. 88-99, 1991. crossref

DRIESSEL, R.; MÖNCH, L. Variable neighborhood search approaches for scheduling jobs on parallel machines with sequence-dependent setup times, precedence constraints, and ready times. Computers \& Industrial Engineering, v. 61 , n. 2 p. 336-345, 2011. crossref

JENSEN, J. B.; PHILIPOOM, P. R.; MALHOTRA, M. K. Evaluation of scheduling rules with commensurate customer priorities in job shops. Journal of Operations Management, v. 13, n. 3, p. 213-228, 1995. crossref

LAMOTHE, J.; MARMIER, F.; DUPUY, M.; GABORIT, P.; DUPONT, L. Scheduling rules to minimize total tardiness in a parallel machine problem with setup and calendar constraints. Computers \& Operations Research, v. 39 , n. 6, p. 1236-1244, 2012. crossref

LAWRENCE, S. R.; SEWELL, E. C. Heuristic Optimal, Static, and Dynamic schedules when processing times are uncertain. Journal of Operations Management, v. 15, n. 1, p. 71-82, 1997. crossref

LEE, Y. H.; BHASKARAN, K.; PINEDO, M. A heuristic to minimize the total weighted tardiness with sequencedependent setups. IIE Transactions, v. 29, n. 1, p. 45-52, 1997. crossref

MLADENOVIC, N.; HANSEN, P. Variable neighborhood search. Computers \& Operations Research, v. 24, n. 11, p. 1097-1100, 1997. crossref

MORTON, T. E.; RACHAMADUGU, R. M. V. Myopic heuristics for the single machine weighted tardiness problem.Working Paper, Pittsburgh: Carnegie Mellon University, 1983. Relatório Técnico.

PAPAVASILEIOU, V.; KOULOURIS, A.; SILETTI, C.; PETRIDES, D. Optimize manufacturing of pharmaceutical products with process simulation and production scheduling tools. Chemical Engineering Research and Design, v. 85 , n. 7, p. 1086-1097, 2007. crossref

PARK, Y.; KIM, S; LEE, Y-H. Scheduling jobs on parallel machines applying neural network and heuristic rules. Computers \& Industrial Engineering, v. 38, n. 1, p. 189-202, 2000. crossref

PFUND, M.; FOWLER, J. W.; GADKARI, A.; CHEN, Y. Scheduling jobs on parallel machines with setup times and ready times. Computers \& Industrial Engineering, v. 54, n. 4, p. 764-782, 2008. crossref

PINEDO, M. L. Scheduling: Theory, Algorithms and Systems. 3 ed. New York: Springer, 2008.

SLACK, N.; CHAMBERS, S.; JOHNSTON, R. Operations Management. 2 ed. São Paulo: Editora Atlas, 2008.

STRASSER, H. Ergonomic efforts aiming at compatibility in work design for realizing preventive occupational health and safety. International Journal of Industrial Ergonomics, v. 16, n. 3, p. 211-235, 1995. crossref

VALENTE, J. M. S; SCHALLER, J. E. Dispatching heuristics for the single machine weighted quadratic tardiness scheduling problem. Computers \& Operations Research, v. 39, n. 9, p. 2223-2231, 2012. crossref

WISNER, J. D.; SIFERD, S. P. A survey of U.S. manufacturing practices in make-to-order machine shops. Production and Inventory Management Journal, v. 36, n. 1, p. 1-7, 1995. 
WOMACK, J. P.; JONES, D. T. A mentalidade enxuta nas empresas: elimine o desperdício e crie riqueza. Rio de Janeiro: Editora Campus, 1996.

XI, Y.; JANG, J. Scheduling jobs on identical parallel machines with unequal future ready time and sequence dependent setup: An experimental study. International Journal of Production Economics, v. 137, n. 1, p. 1-10, 2012. crossref

ZHANG, R.; CHANG, P.-C..; WU, C. A hybrid genetic algorithm for job shop scheduling problem with practical considerations for manufacturing costs: investigations motivated by vehicle production. International Journal of Production Economics, v. 145, n. 1, p. 38-52, 2013. crossref

\section{Dados dos autores:}

Nome completo: Thiago Franzen Aydos

Filiação institucional: Universidade Federal de Santa Catarina

Departamento: POSMEC - Pograma de pós-graduação em Engenharia Mecânica.

Função ou cargo ocupado: Mestrando

Endereço completo para correspondência: Av. Florianópolis 95 / 1004 - Azenha - Porto Alegre.

CEP 90880-460

Telefones para contato: +55 5196320968

e-mail: thiago.aydos@gmail.com

Nome completo: Michel José Anzanello

Filiação institucional: Universidade Federal Rio Grande do Sul

Departamento: Engenharia de Produção e Transportes

Função ou cargo ocupado: Professor

Endereço completo para correspondência: Av. Osvaldo Aranha, 99, 5 andar - Porto Alegre - CEP 90035-190

Telefones para contato: +555196320968

e-mail: michel.anzanello@gmail.com

Submetido: 16/09/2015

Aceito: 16/09/2015 